\title{
Pairing correlations, coherent states, and black holes
}

\section{Document Version}

Accepted author manuscript

Link to publication record in Manchester Research Explorer

\section{Citation for published version (APA):}

Bishop, RF., \& Vourdas, A. (1986). Pairing correlations, coherent states, and black holes. In F. B. Malik (Ed.), Condensed Matter Theories, Vol. 1 (pp. 19-31). Plenum Publishing Corporation.

http://personalpages.manchester.ac.uk/staff/raymond.bishop/RFB_papers/[043] CMT_1(1986)19

\section{Published in:}

Condensed Matter Theories, Vol. 1

\section{Citing this paper}

Please note that where the full-text provided on Manchester Research Explorer is the Author Accepted Manuscript or Proof version this may differ from the final Published version. If citing, it is advised that you check and use the publisher's definitive version.

\section{General rights}

Copyright and moral rights for the publications made accessible in the Research Explorer are retained by the authors and/or other copyright owners and it is a condition of accessing publications that users recognise and abide by the legal requirements associated with these rights.

\section{Takedown policy}

If you believe that this document breaches copyright please refer to the University of Manchester's Takedown Procedures [http://man.ac.uk/04Y6Bo] or contact uml.scholarlycommunications@manchester.ac.uk providing relevant details, so we can investigate your claim.

\section{OPEN ACCESS}




\section{Condensed MATTER THEORIES VOLUME $\mathbf{I}$}

Edited by

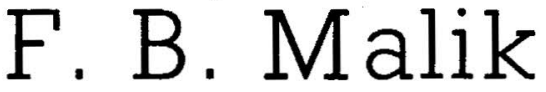

Southern Illinois University at Carbondale Carbondale, Illinois

Plenum Press •New York and London 
Library of Congress Cataloging in Publication Data

International Workshop on Condensed Matter Physics (9th: 1985: San Francisco, Calif.)

Condensed matter theories.

"Proceedings of the Ninth International Workshop on Condensed Matter Physics, held August 5-10, 1985, in San Francisco, California, sponsored by the U.S. Army Research Office at Durham by a grant, and by the San Francisco State University and the Southern Illinois University at Carbondale"-T.p. verso.

Includes bibliographies and index.

1. Condensed matter-Congresses. 2. Quantum liquids-Congresses. I. Malik, F. B. II. San Francisco State University. III. Southern Illinois University at Carbondale. IV. Title.

QC173.4.C65147 1985

$530.4^{\prime} 1$

$86-9400$

ISBN 0-306-42284-0

\section{Conference Organizing Committee}

J. W. Clark (U.S.A.)

M. de Llano (U.S.A.)

F. B. Malik (U.S.A.), Chairperson

C. W. Woo (U.S.A.)

Proceedings of the Ninth International Workshop on Condensed Matter Physics, held August 5-10, 1985, in San Francisco, California, sponsored by the U.S. Army Research Office at Durham by a grant, and by the San Francisco State University and the Southern Illinois University at Carbondale

(C) 1986 Plenum Press, New York

A Division of Plenum Publishing Corporation

233 Spring Street, New York, N.Y. 10013

All rights reserved

No part of this book may be reproduced, stored in a retrieval system, or transmitted in any form or by any means, electronic, mechanical, photocopying, microfilming, recording, or otherwise, without written permission from the Publisher

Printed in the United States of America 
PAIRING CORRELATIONS, COHERENT STATES, AND BLACK HOLES

\author{
R.F. Bishop and A. Vourdas \\ Department of Mathematics \\ University of Manchester Institute \\ of Science and Technology \\ P.0. Box 88 \\ Manchester M60 1QD, England
}

\title{
1. INTRODUCTION
}

Coherent states were first introduced into quantum mechanics by Schrödinger ${ }^{1}$ in 1926, when he explicitly constructed a set of states |A> for the simple harmonic oscillator, which obeyed the classical equations of motion: $\langle\mathrm{A}|\hat{\mathrm{x}}(\mathrm{t})| \mathrm{A}\rangle=\mathrm{x}_{\mathrm{cl}} \equiv \mathrm{x}_{\mathrm{o}} \sin (\omega t+\phi),\langle\mathrm{A}|\hat{\mathrm{p}}(\mathrm{t})| \mathrm{A}\rangle=\mathrm{p}_{\mathrm{cl}} \equiv \mathrm{m \dot {x }} \dot{c l}_{\mathrm{c}} ;$ and which did not display the otherwise rather universal quantum phenomenon of "spreading of the wavepacket" or dispersion. The concept of coherent state as it is understood today was first introduced by Glauber, ${ }^{2}$ who showed how the states constructed by Schrödinger could be used to provide a good quantum-mechanical description of a coherent beam of light as obtained from a laser. Since then, these states have been very profitably further exploited in the field of quantum optics by Glauber and Sudarshan, ${ }^{2,3}$ and many others that followed them.

In order to set the stage for the later developments, some of the properties of these "ordinary" or "atomic" or "Glauber" coherent states are discussed in Section 2. Various of these properties have been used more recently to try to generalize the concept of coherent states. ${ }^{-8}$. Of particular importance here, we mention that various group-theoretical generalizations of coherent states have been given. ${ }^{-7}$ Such coherent states are associated with irreducible representations of various Lie groups. (We should also mention parenthetically, although it is of little consequence for present purposes, that the group-theoretical generalizations are rather divorced from the original motivation of Schrödinger ${ }^{1}$-- namely to discover quantal states that follow the classical motion. Nieto and his co-workers ${ }^{8}$ have sought generalized coherent states which retain this property.)

We adopt the viewpoint that a quantum-mechanical coherent state has the physical property of being non-dispersive and periodic, and the mathematical property of being an element in an orbit in some irreducible space of a Lie group. ${ }^{6,7}$ In this respect, we shall see that the Glauber states are the coherent states of the Heisenberg-Weyl group whose generators, in one dimension, are the boson creation and destruction operators ( ${ }^{\dagger}$ and a respectively) and the identity operator $I$. These three operators together span the Heisenberg-Weyl algebra $W(1):\left[a, a^{\dagger}\right]=I$. 
The physical motivation that now leads us to the particular generalized coherent states that we discuss in Section 3 , is the desire to find a rather general framework in which to discuss pairing correlations in the first instance, and more generally in a later extension, clustering phenomena of more than two particles. Restricting ourșelves to identical bosons, we are led to consider the pairing operators: $a^{\dagger 2}, a^{2}$, and $a^{\dagger} a$. We show in Section 3 that these operators provide a simple realization of the lie algebra $\mathrm{SU}(1,1)$, and we therefore introduce a set of generalized coherent states associated with the corresponding lie group. We should mention that other coherent states of the group $\mathrm{SU}(1,1)$ have been introduced and studied in previous works, $5,6,9$ which have provided hints to their relationship with the Bogoliubov transformation. In the present work we demonstrate this relationship very clearly. In particular we show that simply starting with the idea of pairing operators leads inexorably via the concepts of coherent states to the Bogoliubov transformation itself. Just as the Glauber coherent states may be viewed (and see Section 2) as eigenstates of the original single-boson destruction operator a, we demonstrate that our generalized $\mathrm{SU}(1,1)$ coherent states are also eigenstates of some new single-boson destruction operator $b$, which is itself generated from the operators $a$ and $a^{+}$by a canonical Bogoliubov transformation. In other words, we show how the generalized pairing coherent states may be viewed as ordinary (or Glauber) coherent states of the Bogoliubov quasiparticles. Although they are ordinary coherent states with respect to the operators $\mathrm{b}$ and $\mathrm{b}^{+}$, we also show that they have interesting properties in relation to the original operators a and $a^{\dagger}$, which have not previously received much attention. We believe that these generalized coherent states, and their properties that we discuss here, will be very useful for practical calculations in the many fundamental problems where the Bogoliubov transformation plays a key role - see, for example, Refs. [10-12].

From the motivation already discussed it is apparent that the physical systems to which our results may be applied, will be described by hamiltonians which are at least approximately bilinear in the underlying boson fields. Fairly obvious examples include superfluidity, the parametric excitation of a quantum oscillator, ${ }^{13}$ and the pair production of spinzero particles in a uniform but time-varying electric field. As a rather less obvious example we discuss in Section 4 the application of our results to a uniformly accelerated (Rindler) observer moving in flat (Minkowski) four-space. In particular, if the operators $a$ and $a^{\dagger}$ are now associated with the quanta appropriate, say, to the solutions of the massless KleinGordon equation in the Minkowski metric of an inertial observer, then we show that the operators $b$ and $b^{+}$discussed above may be associated with the corresponding solutions in the so-called Rindler metric appropriate to an observer undergoing uniform proper acceleration. Some previously known results for this important example in relativity can then be rather simply demonstrated using our generalized coherent state results. In particular we may easily show how the Minkowski (inertial) vacuum appears to the Rindler (accelerated) observer as a black-body radiator of quanta with a Planckian (Bose-Einstein) distribution corresponding to a temperature proportional to the proper acceleration. The relationship of this result to the phenomenon of Hawking radiation from black holes ${ }^{14}$ (and hence to the ultimate form of condensed matter:) is pointed out.

\section{THE ORDINARY COHERENT STATES}

The ordinary coherent states are defined with respect to the simple harmonic oscillator with hamiltonian

$$
\mathrm{H}=\mathrm{p}^{2} / 2 \mathrm{~m}+\frac{1}{2} \mathrm{~m} \omega^{2} \mathrm{x}^{2}
$$


The energy eigenstates $|n\rangle$ have eigenergies

$$
E_{n}=\left(n+\frac{1}{2}\right) \hbar \omega,
$$

and are readily given in terms of Hermite polynomials as,

$$
<x \mid n>=\left(\alpha / \pi^{\frac{1}{2}} 2^{n} n !\right)^{+\frac{1}{2}} \exp \left(-\frac{1}{2} \alpha^{2} x^{2}\right) H_{n}(\alpha x), \alpha \equiv(m \omega / \hbar)^{\frac{1}{2}} .
$$

The position and momentum operators can be defined in terms of the annihilation operator $a$ and the creation operator $a^{+}$which is its Hermitian adjoint:

$$
a=\left(\frac{1}{2 m \omega}\right)^{\frac{1}{2}} \hat{p}-i\left(\frac{m \omega}{2 \hbar}\right)^{\frac{1}{2}} \hat{x} .
$$

These operators satisfy the usual boson commutation relation,

$$
\left[a, a^{\dagger}\right]=I \text {, }
$$

and have the property of changing the number of quanta by \pm 1 ,

$$
\begin{aligned}
a \mid n> & =n^{\frac{1}{2}}\left|n-1>, a^{\dagger}\right| n>=(n+1)^{\frac{1}{2}} \mid n+1>, \\
a^{+} a \mid n> & =n|n>,| n>=(n !)^{-\frac{1}{2}}\left(a^{\dagger}\right)^{n} \mid 0>,
\end{aligned}
$$

The ordinary coherent states may now be defined in a number of equivalent ways.

\subsection{Annihilation Operator Coherent States}

The first definition of the ordinary coherent states is that they are eigenstates $|A\rangle$ of the annihilation operator $a$ with complex eigenvalue $A$,

$$
\mathrm{a}|\mathrm{A}>\cdot \mathrm{A}| \mathrm{A}>\quad \cdot
$$

It is then easy to verify, using Eqs. (6), that these states are the particular combinations of the states $\mid \mathrm{n}>$ of definite number of quanta, given by:

$$
\left|A>=\exp \left(-\frac{1}{2}|A|^{2}\right) \sum_{n=0}^{\infty}(n !)^{-\frac{1}{2}} A^{n}\right| n>.
$$

\subsection{Displacement Operator Coherent States}

We have already mentioned in Section 1 that the ordinary coherent states are coherent states of the Heisenberg-Weyl group. A unitary realization of this group is obtained by exponentiating the skew-adjoint operators in the algebra. We are thus led to consider the unitary operators,

$$
\exp \left(A a^{\dagger}-A^{*} a+i \phi\right) ; A \in C, \phi \in \mathbb{R} .
$$

The corresponding unitary representation is then obtained by letting these operators act on a complete set of oscillator states. It is known that this representation is irreducible. By choosing a single representative state from the entire oscillator Hilbert space, and by letting the group elements act on $i t$, we then generate an orbit of the group, whose elements are what a mathematician would call the coherent state of the group. In particular, if we choose the vacuum (ground) state $|0\rangle$ as the representative state, we generate the Glauber or ordinary coherent states. Thus, we define the unitary displacement operator,

$$
U_{1}(A) \equiv \exp \left(A a^{\dagger}-A^{*} a\right)=\exp \left(-\frac{1}{2}|A|^{2}\right) \exp \left(A a^{\dagger}\right) \exp \left(-A^{*} a\right),
$$


for arbitrary complex A. We note that the disp1acement operator is so called because of its action on the operators $a$ and $a^{\dagger}$,

$$
\mathrm{U}_{1}(\mathrm{~A}) \mathrm{aU}_{1}^{\dagger}(\mathrm{A})=\mathrm{a}-\mathrm{A}, \mathrm{U}_{1}(\mathrm{~A}) \mathrm{a}^{\dagger} \mathrm{U}_{1}^{\dagger}(\mathrm{A})=\mathrm{a}^{\dagger}-\mathrm{A}^{*} \text {. }
$$

By displacing the vacuum (ground) state $|0\rangle$ we obtain our second definition of the ordinary coherent state $|A\rangle$ as,

$$
|A\rangle \equiv U_{I}(A)|0\rangle=\exp \left(-\frac{1}{2}|A|^{2}\right) \exp \left(A a^{\dagger}\right)|0\rangle \text {. }
$$

It is trivial to show that the two definitions agree by comparing Eqs. (8) and (11), and making use of Eq. (6).

\subsection{Minimum Uncertainty Coherent States}

A third definition of the ordinary coherent states arises from the Heisenberg position-momentum uncertainty relation,

$$
\Delta x \Delta p \geq \frac{1}{2} \hbar \quad \text {. }
$$

The minimum uncertainty coherent states are then defined both to satisfy the uncertainty relation (12) as an equality and to satisfy a certain subsidiary condition which we shall come to presently. It is a simple exercise in quantum mechanics to show that for any three Hermitian operators A, B and $C$ which satisfy the relation,

$$
[\mathrm{A}, \mathrm{B}]=\mathrm{iC} \text {, }
$$

there is an uncertainty relation,

$$
\Delta \mathrm{A} \triangle \mathrm{B} \geq \frac{1}{2}|<\mathrm{C}>|,
$$

which is satisfied as an equality by solutions $|\psi\rangle$ to the eigenvalue equation,

$$
\Delta B(A-\langle A\rangle)|\psi\rangle=-i \Delta A(B-\langle B\rangle)|\psi\rangle \quad .
$$

Putting $A$ and $B$ respectively equal to $x$ and $p$, and using the uncertainty relation (12) as an equality, this equation is easily solved to give the minimum uncertainty coherent states as:

$$
\langle x \mid \psi\rangle=\left[2 \pi(\Delta x)^{2}\right]^{-\frac{1}{4}} \exp \left\{-\left(\frac{x-\langle x\rangle}{2 \Delta x}\right)^{2}+\frac{i}{\hbar}\langle p\rangle x\right\} .
$$

We can now see that these particular states are defined in terms of the four parameters $\langle x\rangle,\left\langle x^{2}\right\rangle,\langle p\rangle$ and $\left\langle p^{2}\right\rangle$, of which three are so far independent, since the uncertainty relation used as an equality imposes one condition between them. However if one now requires that the oscillator ground state $|0\rangle$ is one of these minimum uncertainty coherent syates, then we clearly require by comparison with Eq. (3) that $\Delta \mathrm{x}$ takes the particular value $\left(2 \alpha^{2}\right)^{-\frac{1}{2}}$ for this state. Since we have already specified the product $\Delta \mathrm{x} \Delta \mathrm{p}=\frac{1}{2} \hbar$ for these states, the subsidiary condition which enables the oscillator ground state to be one of them can equivalently be expressed by fixing the ratio,

$$
\Delta \mathrm{p} / \Delta \mathbf{x}=\mathrm{mw} \quad \text {. }
$$

In this case it is now easy to see that the minimum uncertainty states $|\psi\rangle$ of Eq. (16) subject to the constraint (17) are identical (up to a phase factor) to the annihilation operator uncertainty states $|A\rangle$ of Eq. (8). We do this by writing the defining equation (7) for the states $|A\rangle$ as, 


$$
\begin{aligned}
& \frac{1}{2}\left(\frac{\hat{p}}{\Delta \mathrm{p}}-i \frac{\hat{\mathrm{x}}}{\Delta \mathrm{x}}\right)|\mathrm{A}\rangle=\mathrm{A}|\mathrm{A}\rangle \equiv \frac{1}{2}\left(\frac{\langle\mathrm{p}\rangle}{\Delta \mathrm{p}}-\mathrm{i} \frac{\langle\mathrm{x}\rangle}{\Delta \mathrm{x}}\right)|\mathrm{A}\rangle, \\
& \Delta \mathrm{p} \equiv(\mathrm{m} \hbar \omega / 2)^{\frac{1}{2}}, \quad \Delta \mathrm{x} \equiv(\hbar / 2 \mathrm{~m} \omega)^{\frac{1}{2}}
\end{aligned}
$$

where we have used on1y Eq. (4). We then note firstly that the values $\Delta p$ and $\Delta \mathrm{x}$ of $\mathrm{Eq}$. (18) are precisely those obtained from the minimum uncertainty condition (12) and the subsidiary condition (17); and secondly that the differential equation (18) is now identical to the equation (15) for $|\psi\rangle$ when $\mathrm{A} \rightarrow \mathrm{x}, \mathrm{B} \rightarrow \mathrm{p}$.

We have thus shown that these three definitions of the ordinary coherent states are all equivalent. These states, as is by now well known, have a number of characteristic properties which will be useful later. In the first place it is easy to show that they are not mutually orthogonal,

$$
\langle D \mid A\rangle=\exp \left[-\frac{1}{2}\left(|A|^{2}+|D|^{2}-2 D * A\right)\right],|\langle D \mid A\rangle|^{2}=\exp \left(-|D-A|^{2}\right) .
$$

Secondly, they form an overcomplete basis, in the sense that they contain more states than necessary for the expansion of an arbitrary state. It is also possible to give a very useful resolution of the identity operator in terms of them. Thus, consider the operator $J$ defined as,

$$
\mathrm{J} \equiv \int \mathrm{d}^{2} \mathrm{~A} \mid \mathrm{A}>\langle\mathrm{A}| ; \mathrm{d}^{2} \mathrm{~A} \equiv \mathrm{d}(\operatorname{Re} \mathrm{A}) \mathrm{d}(\operatorname{Im} \mathrm{A}),
$$

where the integration extends over the entire complex-A plane. It is not difficult to show that all operators $U_{1}(B)$, defined in Eq. (9), commute with the operator $J$, by using the definition (11) and the group property

$$
\mathrm{U}_{1}(A) \mathrm{U}_{1}(\mathrm{~B})=\exp \left[\mathrm{i} \operatorname{Im}\left(A \mathrm{~B}^{*}\right)\right] \mathrm{U}_{1}(\mathrm{~A}+\mathrm{B}) \text {, }
$$

which is readily proven. Since the operators $U_{1}(A) \exp (i \phi)$ thus form a group (namely the Heisenberg-Weyl group), Schur's lemma immediately gives that $\mathrm{J}$ is a constant multiple of the identity operator. By taking the expectation value of $\mathrm{J}$ in any state $|\mathrm{B}\rangle$, and using the orthogonality relation (19), this constant is readily evaluated, and we find that the identity can be expanded in terms of the ordinary coherent states as,

$$
\frac{1}{\pi} \int \mathrm{d}^{2} \mathrm{~A}|\mathrm{~A}><\mathrm{A}|=\mathrm{I} \text {. }
$$

From the preceding discussion it is clear that the ordinary coherent states should be a useful tool whenever the Heisenberg-Weyl group is a dynamical symmetry of the hamiltonian. In such cases the Heisenberg equations of motion simply become equivalent to the corresponding equations for the classical quantities. The coherent states then remain coherent as time evolves, and the motion in the complex-A plane of the point corresponding to a particular coherent state is just described by its classical path. In such cases one has exactly reduced a quantum problem to a classical problem. An obvious question at this point is whether any of this procedure, and in particular whether any of our definitions of ordinary coherent states, can be generalized to other dynamical symmetry groups. As we asserted already in Section 1, group-theoretical generalizations based both on the annihilation operator definition ${ }^{5}$ and on the displacement operator definition, ${ }^{6}$ have been given. Our particular motivation to proceed further is now the possibility to understand pairing correlations in a similar fashion.

\section{PAIRING OPERATORS AND THE SU $(1,1)$ COHERENT STATES}

In connection with pairing (of bosons), we naturally consider the 
operators $a^{\dagger}, a^{2}, a^{\dagger} a$ and $a^{\dagger}$. It is trivial to show that these operators are again closed under commutation. More specifically, the three operators

$$
\mathrm{K}_{+} \equiv \frac{1}{2} \mathrm{a}^{\dagger} 2, \mathrm{~K}_{-} \equiv \frac{1}{2} \mathrm{a}^{2}, \mathrm{~K}_{0} \equiv \frac{1}{2} \mathrm{a}^{\dagger} \mathrm{a}+\frac{1}{4}
$$

are readily seen to satisfy the Lie algebra of $\mathrm{SU}(1,1)$, namely

$$
\left[\mathrm{K}_{0}, \mathrm{~K}_{ \pm}\right]= \pm \mathrm{K}_{ \pm} ;\left[\mathrm{K}_{-}, \mathrm{K}_{+}\right]=2 \mathrm{~K}_{\mathrm{o}} \text {. }
$$

We note that $S U(1,1)$ is locally isomorphic to the group $S O(2,1)$ of rotations in three-dimensional (2 space +1 time) Minkowski space. The algebra (24) may be compared with the more familiar Lie algebra of SU(2),

$$
\left[\mathrm{J}_{0}, \mathrm{~J}_{ \pm}\right]= \pm \mathrm{J}_{ \pm} ;\left[\mathrm{J}_{-}, \mathrm{J}_{+}\right]=-2 \mathrm{~J}_{0},
$$

which is itself locally isomorphic to the group So(3) of rotations in threedimensional Euclidean space. Just as in Section 2.2, a unitary realization of the group $\mathrm{SU}(1,1)$ may be obtained by exponentiating the skew-adjoint operators in the algebra. We are thus led to introduce the unitary operators,

$$
\begin{aligned}
U_{2}(\rho, \theta, \lambda) & \equiv \exp \left(-\frac{1}{4} \rho \mathrm{e}^{-i \theta} \mathrm{a}^{\dagger}{ }^{+}+\frac{1}{4} \rho \mathrm{e}^{i \theta} \mathrm{a}^{2}\right) \exp \left(i \lambda \mathrm{a}^{\dagger} \mathrm{a}\right) ; \rho, \theta, \lambda \in \mathbb{R} \\
\mathrm{U}_{2}^{\dagger} \mathrm{U} 2 & =\mathrm{I} .
\end{aligned}
$$

Using this definition, it is not difficult to show that the mode of action of the operators $U_{2}$ on the creation and destruction operators is given by the relations,

$$
\begin{aligned}
& \mathrm{U}_{2}(\rho, \theta, \lambda) \mathrm{aU}_{2}^{\dagger}(\rho, \theta, \lambda)=e^{-i \lambda}\left[\cosh \left(\frac{1}{2} \rho\right) a+e^{-i \theta} \sinh \left(\frac{1}{2} \rho\right) a^{\dagger}\right] \equiv \mathbf{b}, \\
& \mathrm{U}_{2}(\rho, \theta, \lambda) \mathrm{a}^{+} \mathrm{U}_{2}^{\dagger}(\rho, \theta, \lambda)=e^{i \lambda}\left[\cosh \left(\frac{1}{2} \rho\right) \mathrm{a}^{+}+\mathrm{e}^{i \theta} \sinh \left(\frac{1}{2} \rho\right) \mathrm{a}\right] \equiv \mathrm{b}^{\dagger},
\end{aligned}
$$

which are the analogues of Eqs. (10) for the (Heisenberg-Wey1) displacement operators. We see that the transformation (27) imposed by the operators $\mathrm{U}_{2}$ on the boson operators $\mathrm{a}$ and $\mathrm{a}^{+}$is just the usual Bogoliubov transformation to new boson operators $b$ and $b^{\dagger}$ that still satisfy the boson commutation relations

$$
\left[\mathrm{b}, \mathrm{b}^{\dagger}\right]=\mathrm{I} \text {. }
$$

Furthermore, from Eqs. (27) and the fact that $U_{2}$ is unitary, we can trivially prove for any function $f\left(a, a^{+}\right)$that can be expanded in a power series in its arguments, the relation,

$$
\mathrm{U}_{2} \mathrm{f}\left(\mathrm{a}, \mathrm{a}^{\dagger}\right) \mathrm{U}_{2}^{\dagger}=\mathrm{f}\left(\mathrm{b}, \mathrm{b}^{\dagger}\right) \longleftrightarrow \mathrm{U}_{2} \mathrm{f}\left(\mathrm{a}, \mathrm{a}^{\dagger}\right)=\mathrm{f}\left(\mathrm{b}, \mathrm{b}^{\dagger}\right) \mathrm{U}_{2} \text {. }
$$

In terms of the operators $U_{2}$, we now introduce the states $|A ; \rho \theta \lambda\rangle$ defined as,

$$
|A ; \rho \theta \lambda\rangle \equiv U_{2}(\rho, \theta, \lambda)|A\rangle=U_{2}(\rho, \theta, \lambda) U_{1}(A)|0\rangle,
$$

which are now our prime objects of study as generalized coherent states. We stress immediately that the representative state that has now been chosen for $U_{2}$ to act on, and so to generate an orbit of the group $\operatorname{SU}(1,1)$, is just an ordinary coherent state $\mid A>$. We also note from Eq. (7) that this state $|A\rangle$ is an eigenstate of the operator $K_{-}$,

$$
K_{-}|A\rangle=\frac{1}{2} A^{2}|A\rangle \text {, }
$$

but not of the operator $\mathrm{K}_{0}$. We stress this point only since in the literature, different SU $(1,1)$ coherent states are often defined by letting $U_{2}$ 
(or a similar operator) act on an eigenstate of $\mathrm{K}$, and in doing this much of the underlying simplicity is obscured. We also note that Eq. (29) immediately gives that $\mathrm{U}_{2} \mathrm{a}=\mathrm{bU}_{2}$, and hence that the states $|\mathrm{A} ; \rho \theta \lambda\rangle$ are eigenstates of the destruction operator $b$,

$$
\mathrm{b}|\mathrm{A} ; \rho \theta \lambda\rangle=\mathrm{bU}_{2}(\rho, \theta, \lambda)|\mathrm{A}\rangle=\mathrm{U}_{2}(\rho, \theta, \lambda) \mathrm{a}|\mathrm{A}\rangle=\mathrm{A}|\mathrm{A} ; \rho \theta \lambda\rangle,
$$

by making use of Eq. (7).

The special case $A=0$ is of particular interest since Eq. (32) implies the relation

$$
b|0 ; \rho \theta\rangle=0,
$$

where we have put $|0 ; p \theta \lambda\rangle \equiv|0 ; p \theta\rangle$ since Eqs. (6) and (26) show that this state is independent of $\lambda$. Thus, just as the state $|0\rangle$ was defined by the relation $a \mid 0>=0$ to be the ground state with respect to the operators $a$ and $a^{\dagger}$ (i.e., the vacuum for a-type bosons), so the state $|0 ; p \theta\rangle \equiv$ $\mathrm{U}_{2}(\rho, \theta, \lambda) \mid 0>$ obeys Eq. (33), and is thus the vacuum for b-type bosons. Using Eq. (29) again we find,

$$
\mathrm{U}_{2}(\rho, \theta, \lambda) \exp \left(A a^{\dagger}-A^{*} a\right)=\exp \left(A b^{\dagger}-A^{*} b\right) U_{2}(\rho, \theta, \lambda),
$$

and hence from Eqs. (9), (11) and (30),

$$
|A ; \rho \theta \lambda\rangle=\exp \left(\mathrm{Ab}^{\dagger}-\mathrm{A}^{*} \mathrm{~b}\right)|0 ; \rho \theta\rangle \text {. }
$$

In this way, we see very clearly that the states $|A ; \rho \theta \lambda\rangle$ may be viewed as ordinary coherent states with respect to the operators $b$ and $b^{\dagger}$. In other words, they are the Glauber coherent states for the Bogoliubov quasiparticles, or b-type bosons. Before proceeding, we also note at this point a particularly important relation for future use, for the expectation value of the number of b-type bosons in the vacuum $|0\rangle$ for a-type bosons. This quantity is immediately obtained from the definition of Eq. (27) as,

$$
\left\langle 0\left|b^{\dagger} b\right| 0\right\rangle=\sinh ^{2}\left(\frac{1}{2} \rho\right) \text {. }
$$

Explicit evaluation of the generalized coherent states of Eq. (30) is not particularly easy with $\mathrm{U}_{2}$ given by its defining equation (26). This is made much easier by making use of the 'norma1-ordering relation',

$$
\begin{aligned}
& \exp \left(-\frac{1}{2} \rho e^{-i \theta} K_{+}+\frac{1}{2} \rho e^{i \theta} K_{-}\right)=e^{\sigma K_{+}} e^{\tau K_{o}} e^{-\sigma * K_{-}}, \\
& \sigma \equiv-e^{-i \theta} \tanh \left(\frac{1}{2} \rho\right), \tau \equiv \ln \left(1-|\sigma|^{2}\right),
\end{aligned}
$$

which has been given by Perelomov, ${ }^{6}$ and which is valid for any operators $\mathrm{K}_{+}, \mathrm{K}_{-}$and $\mathrm{K}_{\mathrm{O}}$ which satisfy the $\mathrm{SU}(1,1)$ algebra of $\mathrm{Eq} .(24)$. We note parenthetically that it is simplest, and sufficient, to prove Eq. (37) for the most elementary representation $K_{0} \rightarrow \frac{1}{2} \sigma_{3}, k_{ \pm} \rightarrow \frac{1}{2} i\left(\sigma_{1} \pm i \sigma_{2}\right)$ of the algebra, in terms of the usual Pauli spin matrices $\sigma_{1}, \sigma_{2}$ and $\sigma_{3}$ which obey the SU(2) algebra of Eq. (25). Using the result of ${ }^{2} \mathrm{Eq}$. (37), we may write the operator $U_{2}(\rho, \theta, \lambda)$ of Eq. (26) in the equivalent form:

$$
\begin{aligned}
\mathrm{U}_{2}(\rho, \theta, \lambda) & \equiv \mathrm{U}_{2}(\sigma, \lambda) \\
& =\exp \left(\frac{1}{2} \sigma \mathrm{a}^{\dagger}\right)\left(1-|\sigma|^{2}\right)^{\frac{1}{2} a^{\dagger} a+\frac{1}{4}} \exp \left(-\frac{1}{2} \sigma * a^{2}\right) \exp \left(i \lambda a^{\dagger} a\right),
\end{aligned}
$$

where $\lambda$ is real and $\sigma$, given by Eq. (37) is complex and with modulus $|\sigma|<1$. Similarly, by making trivial use of Eqs. (6) and (8) we can first derive the relation, 


$$
\exp \left(\phi \mathrm{a}^{\dagger} \mathrm{a}\right)|\mathrm{A}\rangle=\exp \left[\frac{1}{2}|\mathrm{~A}|^{2}\left(\mathrm{e}^{\phi+\phi^{*}}-1\right)\right] \mid A \mathrm{e}^{\phi}>,
$$

which is valid for any complex $\phi$, and then use this relation to write the generalized coherent states of Eq. (30) in the equivalent form,

$$
\begin{aligned}
\mid A ; \rho \theta \lambda> & \left.\equiv\left|A ; \sigma \lambda>=U_{2}(\sigma, \lambda)\right| A\right\rangle \\
& =\left(1-|\sigma|^{2}\right)^{\frac{1}{4}} \exp \left(-\frac{1}{2} \sigma * A^{2} e^{2 i \lambda}-\frac{1}{2}|\sigma|^{2}|A|^{2}\right) \exp \left(\frac{1}{2} \sigma a^{+2}\right)\left|A e^{i \lambda}\left(1-|\sigma|^{2}\right)^{\frac{1}{2}}\right\rangle .
\end{aligned}
$$

In the special case $\lambda=0=\sigma$ we simply regain the ordinary coherent states: $|A ; 00\rangle=|A\rangle$. We also note again that when $A=0$, the $b$-type vacuum $|0 ; \sigma \lambda\rangle \equiv|0 ; \sigma\rangle$ is independent of $\lambda$.

Since, as we have seen, the states $|A ; \sigma \lambda\rangle$ may simply be regarded as ordinary coherent states of the operators $b$ and $b^{+}$, it is clear that for fixed values of $\sigma$ and $\lambda$ they therefore obey all of the usual properties of coherent states. For example, the analogues of Eqs. (19) and (22) are respectively,

$$
\begin{aligned}
& \langle\mathrm{D} ; \sigma \lambda \mid \mathrm{A} ; \sigma \lambda\rangle=\exp \left[-\frac{1}{2}\left(|\mathrm{~A}|^{2}+|\mathrm{D}|^{2}-2 \mathrm{D} * \mathrm{~A}\right)\right] ; \\
& \frac{1}{\pi} \int \mathrm{d}^{2} \mathrm{~A}|\mathrm{~A} ; \sigma \lambda\rangle\langle\mathrm{A} ; \sigma \lambda|=\mathrm{I} .
\end{aligned}
$$

Just as in Eq. (8), the states $|A ; \sigma \lambda\rangle$ may also be expressed in terms of the eigenstates $|\mathrm{n} ; \sigma \lambda\rangle$ of the number operator $\mathrm{b}^{\dagger} \mathrm{b}$ for $\mathrm{b}$-type bosons (and compare with Eq. (6)),

$$
\mathrm{b}^{\dagger} \mathrm{b}|\mathrm{n} ; \sigma \lambda\rangle=\mathrm{n}|\mathrm{n} ; \sigma \lambda\rangle,|\mathrm{n} ; \sigma \lambda\rangle=(\mathrm{n} !)^{-\frac{1}{2}}\left(\mathrm{~b}^{\dagger}\right)^{\mathrm{n}}|0 ; \sigma\rangle,
$$

in the form,

$$
\left|A ; \sigma \lambda>=\exp \left(-\frac{1}{2}|A|^{2}\right) \sum_{n=0}^{\infty}(n !)^{-\frac{1}{2}} A^{n}\right| n ; \sigma \lambda>.
$$

For some purposes it is also useful to compare the ordinary coherent states $|A\rangle$ (with $\sigma=0=\lambda$ ) with our generalized coherent states $|B ; \sigma \lambda\rangle$ in the following way. Use of Eqs. (40), (7) and (19) gives the overlap,

$$
\begin{aligned}
\langle A \mid B ; \sigma \lambda\rangle=\left(1-|\sigma|^{2}\right)^{\frac{1}{4}} \exp [ & -\frac{1}{2}|A|^{2}-\frac{1}{2}|B|^{2}+\frac{1}{2} \sigma A *^{2} \\
& \left.-\frac{1}{2} \sigma * B^{2} e^{2 i \lambda}+A * B e^{i \lambda}\left(1-|\sigma|^{2}\right)^{\frac{1}{2}}\right] .
\end{aligned}
$$

We observe that although the states $|A\rangle$ and $|B ; \sigma \lambda\rangle$ are not generally orthogonal, they become so in the limit that $|\sigma|$ approaches unity. Finally, use of the completeness relation (22), yieIds the expansion

$$
|B ; \sigma \lambda\rangle=\frac{1}{\pi} \int d^{2} A\langle A \mid B ; \sigma \lambda\rangle|A\rangle
$$

for the generalized coherent states in terms of the Glauber coherent states, and where the overlap integrals $\langle A \mid B ; \sigma \lambda\rangle$ are given explicitly in Eq. (45). A special case of Eqs. (45) and (46) of particular interest is the resolution of the vacuum for b-type bosons,

$$
|0 ; \sigma\rangle=\frac{1}{\pi}\left(1-|\sigma|^{2}\right)^{\frac{1}{4}} \int \mathrm{d}^{2} \mathrm{~A} \exp \left(-\frac{1}{2}|\mathrm{~A}|^{2}+\frac{1}{2} \sigma \mathrm{A}^{* 2}\right)|\mathrm{A}\rangle .
$$

We now illustrate the use of our generalized SU $(1,1)$ coherent states and their associated Bogoliubov transformation, with an example drawn fron relativistic quantum field theory. 


\section{A POOR MAN'S BLACK HOLE AND HAWKING RADIATION}

In the relativistic case of flat (Minkowski) four-space, it has been known for some time $15-18$ that a Bogoliubov transformation analogous to Eq. (27) may be associated with a uniformly accelerated observer moving through the space. Since in many ways this particular example models the behaviour of real black holes, we shall show how our results of Section 3 can now be given a particularly interesting physical interpretation.

In terms of the usual Minkowski time-space coordinates $(t, x, y, z)$, in units with $c=1$, the inertial (or Minkowski) observer sees the flat spacetime line element

$$
d s^{2}=-d t^{2}+d x^{2}+d y^{2}+d z^{2} \quad .
$$

One well-known way to introduce a 'horizon' into this space (and hence to model a black hole) is by the transformation to Rindler coordinates $(\tau, \xi, y, z)$,

$$
\begin{aligned}
& x=\xi \cosh \tau \\
& t=\xi \sinh \tau
\end{aligned} ; \tau, \xi \in \mathbb{R}(-\infty, \infty) \Longleftrightarrow \begin{aligned}
& \xi= \pm\left(x^{2}-t^{2}\right)^{\frac{1}{2}} \\
& \tau=\tanh ^{-1}(t / x) .
\end{aligned}
$$

We note that this transformation maps only the regions that we now call the right $(x>|t|)$ and left $(x<-|t|)$ 'Rindler wedges' respectively. The forward $(t>|x|)$ and backward $(t<-|x|)$ light cones are unmapped, and the lines $x= \pm t$ (or equivalently $\xi=0, \tau \rightarrow \pm \infty$ ) have become horizons to the Rindler observer. The line element (48) is mapped by the transformation (49) into

$$
d s^{2}=-\xi^{2} d \tau^{2}+d \xi^{2}+d y^{2}+d z^{2} .
$$

One sees that lines of constant $\xi$ correspond to world-1ines of so-called Rindler observers who undergo uniform proper acceleration $\xi^{-1}$ and who see proper time elements $\xi d \tau$.

Our aim is now to do quantum field theory in the Rindler coordinates. For simplicity, we work with a massless scalar (boson) field $\phi$, which hence obeys the massless Klein-Gordon (or scalar wave) equation,

$$
-\frac{\partial^{2} \phi}{\partial t^{2}}+\nabla^{2} \phi=0 \Longleftrightarrow-\frac{1}{\xi^{2}} \frac{\partial^{2} \phi}{\partial \tau^{2}}+\frac{\partial^{2} \phi}{\partial \xi^{2}}+\frac{1}{\xi} \frac{\partial \phi}{\partial \xi}+\frac{\partial^{2} \phi}{\partial y^{2}}+\frac{\partial^{2} \phi}{\partial z^{2}}=0 .
$$

It is easy to show by separation of variables that a complete set of solutions to the classical wave equation (51) in Rindler coordinates is given (for $|x|>|t|$ ) by,

$$
\begin{aligned}
u_{\omega \vec{k}}(\tau \xi y z) & =N_{\omega \vec{k}} \exp \left(-i \omega \tau+i k_{2} y+i k_{3} z\right) k_{i \omega}(k \xi) ; \\
\vec{k} & \equiv\left(k_{2}, k_{3}\right) ; k=|\vec{k}|
\end{aligned}
$$

where $\mathrm{N}_{\omega \overrightarrow{\mathrm{k}}}$ is a normalization constant and $\mathrm{K}_{\mathrm{i} \omega}(\rho)$ is a solution to the modified Bessel equation,

$$
\rho^{2} \frac{d^{2} X}{d \rho^{2}}+\rho \frac{d X}{d \rho}+\left(\omega^{2}-\rho^{2}\right) x=0 .
$$

A technical point to note here is that whereas in the right Rindler wedge $(x>|t|)$ the solutions $u_{\omega \vec{k}}$ and $u_{\omega \vec{k}}^{*}$ represent respectively the positive and negative frequency modes with respect to the operator $i \partial / \partial \tau$, the converse is true in the left Rindler wedge $(x<-|t|)$. The basic reason for this is that in the right Rindler wedge the direction of increasing $\tau$ 
on lines of constant $\xi$ is in the direction of increasing $t$, whereas in the left Rindler wedge the direction is opposite. Bearing this in mind, quantization of the massless scalar field in Rindler coordinates is now based on the usual expansion in canonical modes,

$$
\phi(\tau \xi y z)=\int_{0}^{\infty} d \omega \int \frac{d^{2} \vec{k}}{(2 \pi)^{2}}\left[u_{\omega \vec{k}}^{(+)} b_{\omega \vec{k}}^{(+)}+u_{\omega \vec{k}}^{(-)} b_{\omega \vec{k}}^{(-)}+h \cdot c \cdot\right],
$$

where h.c. indicates the hermitian conjugate; the functions $u \stackrel{(+)}{u}$ and $u(\vec{r})$ are solutions to the wave equation which vanish respectively in the left and right Rindler wedges but which equal $u \vec{u}$ in the opposite Rindler wedge; and the operators $\mathrm{b}(\stackrel{(q)}{\omega \vec{k}}$ obey the usual canonical commutation relations,

$$
\left[{ }^{b} \underset{\omega \vec{k}}{(\sigma)}, b_{\omega^{\prime} \vec{k}^{\prime}}^{\left(\sigma^{\prime}\right)}\right]=\delta_{\sigma \sigma^{\prime}}, \delta\left(\omega-\omega^{\prime}\right) \delta\left(\vec{k}-\vec{k}^{\prime}\right) ; \sigma= \pm .
$$

We now note that the solutions $\stackrel{(\stackrel{ \pm}{\vec{t}})}{\vec{u}}$ are analytic everywhere except on the horizons $x \pm t=0$. Indeed it ${ }^{\omega k}$ is not difficult to show from Eqs. (52) and (53) that on these horizons, $u \vec{k}$ behaves like a regular

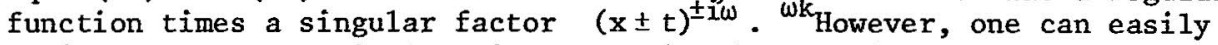
verify that the particular linear combinations defined by

$$
v_{\omega \vec{k}}^{( \pm)} \equiv[1-\exp (-2 \pi \omega)]^{-\frac{1}{2}}\left[u \stackrel{( \pm)}{\omega \vec{k}}+\exp (-\pi \omega) u_{\omega \vec{k}}^{(\vec{f})}\right],
$$

are also analytic on the horizons $x=t$ and $x=-t$ respectively, and indeed are analytic respectively in the lower half complex $(t-x)$ plane or the upper half complex $(t+x)$ plane. This fact can then be used to show that the solutions $v^{\left(\frac{f}{\vec{l}}\right)}$ may therefore be used for a Minkowski decomposition of the field $\$$ for the jnertial observer, and also that in this case the solutions $v(+)$ and $v \vec{b}$ are respectively the positive and negative frequency modes with $\mathrm{wk}_{\mathrm{k}}$ spect to the operator $i \partial / \partial t$. The Minkowski decomposition analogous to Eq. (54) is therefore

$$
\phi(\text { txyz })=\int_{0}^{\infty} \mathrm{d} \omega \int \frac{\mathrm{d}^{2} \overrightarrow{\mathrm{k}}}{(2 \pi)^{2}}\left[\begin{array}{c}
(+) \\
\mathrm{w}^{\mathrm{k}}
\end{array}\right.
$$
where the operators a $\underset{( \pm)}{\vec{k}}$ obey canonical boson commutation relations ana-
logous to Eq. (55).

A comparison of the two decompositions (54) and (57), together with Eq. (56), easily yields the relation,

$$
\mathrm{b}_{\omega \overrightarrow{\mathrm{k}}}^{( \pm)}=\left(1-\mathrm{e}^{-2 \pi \omega}\right)^{-\frac{1}{2}}\left[\mathrm{a}^{( \pm \overrightarrow{\mathrm{k}}}+\mathrm{e}^{-\pi \omega} \mathrm{a}_{\omega \overrightarrow{\mathrm{k}}}^{(\mp)+}\right] \text {, }
$$

between the boson operators in the two representations. Defining new operators,

$$
a_{\omega \vec{k}} \equiv e^{i \gamma}(\underset{\omega \vec{k}}{a(+)}+\underset{\omega \vec{k}}{a(-)}), \quad b_{\omega \vec{k}} \equiv e^{i \gamma}(\underset{\omega \vec{k}}{b \underset{(+)}{(\vec{k}}}+\underset{\omega \vec{k}}{(-)}),
$$

where $\gamma$ is an arbitrary real constant, we find that the relationship between them,

$$
b_{\omega \vec{k}}=\left(1-e^{-2 \pi \omega}\right)^{-\frac{1}{2}}\left[a_{\omega \vec{k}}+\exp (-\pi \omega+2 i \gamma) a_{\omega \vec{k}}^{+}\right],
$$

is precisely the Bogoliubov transformation (27) with the identification,

$$
\tanh \left(\frac{1}{2} p\right)=e^{-\pi \omega}
$$


It is therefore clear that all of our general results may now be applied to this example. In particular, the states $|0\rangle$ and $|0 ; \sigma\rangle$ now play the role of vacua for our massless scalar meson field as observed respectively by the inertial (Minkowski) and uniformly accelerated (Rindler) observers. In particular, a direct combination of Eqs. (36) and (61) gives the very important result that the inertial vacuum $\mid 0>$ appears to the uniformly accelerated observer, for whom the meson number operator is $\vec{b} \vec{k} \vec{b} \vec{k}$, as a source of mesons with distribution,

$$
\left\langle 0\left|b_{\omega \vec{k}}^{\dagger} b_{\omega \vec{k}}\right| 0\right\rangle=\left(e^{2 \pi \omega}-1\right)^{-1} .
$$

We recall that along lines of constant $\xi$, proper time is marked by the product $\xi \tau$. Hence the Rindler observer interprets a wave varying as $\exp (-i \omega \tau) \equiv \exp [-i(\omega / \xi) \xi \tau]$, as in Eq. (52), as having an angular frequency $w=\omega / \xi$. Comparing Eq. (62) with a black-body radiator, which at temperature $T$ has the usual Planckian distribution $\left[\exp \left(\hbar w / k_{B} T\right)-1\right]^{-1}$ for massless bosons, yields the profound result that the Minkowski vacuum appears to the uniformly accelerated observer as a black-body radiator (at least for massless scalar mesons) of temperature given by,

$$
T=\frac{\hbar}{2 \pi k_{B} c \xi},
$$

where we have re-inserted the correct factors of c. Davies ${ }^{15}$ seems to have been the first to realize that the Rindler vacuum contains a thermal distribution of quanta relative to the Minkowski vacuum. We note from Eq. (63) that the effect is explicitly both quantum-mechanical and relativistic in origin since $T$ vanishes in both limits $\hbar \rightarrow 0$ and $c \rightarrow \infty$. We note also that the temperature is directly proportional to the proper acceleration $\xi^{-1}$.

From our derivation it is clear that the underlying 'cause' of these results is the existence, in the Rindler coordinates, of the horizons at $x= \pm t$. Sciama et al. ${ }^{18}$ have stressed how an observer undergoing uniform acceleration may thus profitably be viewed as constituting a "model" or "poor man's" black hole. They have further shown the deep connection between our present result and the thermal properties of real black holes when quantum effects are taken into account. From our own derivation it is no surprise that our result is essentially a poor man's description of Hawking radiation from real black holes, which have been shown ${ }^{14}$ to behave as black-body radiators with temperature given as in Eq. (63) but with the proper acceleration $\xi^{-1}$ now replaced by the so-called "surface (acceleration due to) gravity".

\section{CONCLUSIONS}

We have investigated in this paper the generalized coherent states $|A ; \sigma \lambda\rangle=U_{2}(\sigma, \lambda)|A\rangle$ obtained from the normal or Glauber coherent states $|A\rangle=U_{1}(A)|0\rangle$. The coherence properties of the Glauber states $|A\rangle$ are already contained in the exponentiation of the one-body operator which defines the displacement operator $\mathrm{U}_{1}$, where by $_{+}$a one-body operator we mean one linear in the field operators a and $a^{t}$. Similarfy the operator $\mathrm{U}_{2}$ contains exponentials of operators bilinear in $\mathrm{a}$ and $\mathrm{a}$, and was thus expected to generate a coherent paired state. We have shown how this generalized coherent state can itself be alternatively viewed as a normal coherent state with respect to new boson (quasiparticle) operators that stand in relation to the original boson (particle) operators by a Bogoliubov transformation. We showed in particular how the Bogoliubov transformation itself arises most naturally in our framework, starting only with the basic 
concept of building coherent paired states. In this respect a number of generalizations immediately suggest themselves.

Firstly, the extension from a single boson (or canonical quantum mode) to a set of $\mathrm{n}$ distinct bosons (or modes) is possible. In the case of a single boson treated here, we saw that there is an underlying lie group, namely $\mathrm{SU}(1,1)$ associated with the generalized coherent states. In fact this is not the only appopriate group here, since the three-dimensional Lorentz group $\mathrm{SO}(2,1)$ is not only locally isomorphic to $\mathrm{SU}(1,1)$ but also to both the group SL $(2, R)$ of real second order matrices with unit determinant and the symplectic group $S p(2, R)$. Similarly the bilinear products of boson operators $a_{i} a_{j}, a_{i}^{\dagger} a_{j}$ and $a_{i} a_{j}$ for $i, j=1, \cdots, n$, also form a basis for a realization of the higher symplectic algebra $S p(2 n, R)$. $A$ unitary realization of this group ean similarly be constructed by exponentiating the skew-adjoint operators in this algebra, and it should then be possible to extend in an obvious way the treatment given here to the case of $\mathrm{n}$ distinct bosons.

A second generalization concerns the possibility of looking at higher clustering correlations in the same way. Thus, for correlated clusters of $m$ identical bosons, we may envisage taking the various products of order $m$ in the operators $a$ and $a^{+}$, examining their underlying group structure, and so constructing the appropriate coherent states. In this way one can hope to generalize the Bogoliubov transformation appropriate for (a particular type of) pairing, to transformations appropriate for generating higher clustering and the associated possible new condensed phases. A third possible extension is to repeat the above for clustering correlations in systems of fermions. We are presently interested in each of these extensions.

We have already mentioned in Section 1 various possible physical applications of the pairing coherent states discussed here, and we have illustrated our results in Section 4 in a context perhaps not familiar to condensed matter theorists. We started our discussion in Section 1 with the work of Glauber and others on the ordinary coherent states in the field of quantum optics, and it is perhaps appropriate to return finally to this field. Just as the Glauber states are appropriate for a description of the one-photon coherent states and hence the radiation field from a conventional single-photon laser, so our generalized $\mathrm{SU}(1,1)$ paired coherent states have been discussed in quantum optics 10,11 from the viewpoint of two-photon coherent states and the possibility of a two-photon laser. In quantum optics the two-photon coherent states have been called "squeezed states", since although they are evidently minimum uncertainty states in the sense of realizing the uncertainty relation (12) as an equality, it is not difficult to show that the ratio $\Delta \mathrm{p} / \Delta \mathrm{x}$ for the generalized coherent state is "squeezed" in the sense that it is not the value of $\mathrm{Eq}$. (17) for ordinary coherent states, but acquires an extra factor $(1+\sigma) /(1-\sigma)$. There is much present work and excitement in quantum optics on these states. It is our belief that the possible extensions discussed above will have immediate repercussions not only in condensed matter theory but also in quantum optics.

Acknowledgement

We gratefully acknowledge support for this work in the form of a Research Grant from the Science and Engineering Research Council of Great Britain. 
1. E. Schrödinger, Naturwiss . 14:664 (1926).

2. R. J. Glauber, Phys. Rev. Lett. 10:84 (1963); Phys. Rev. 130:2529 (1963) and $131: 2766$ (1963).

3. E. C. G. Sudarshan, Phys. Rev, Lett. 10:227 (1963);

R. J. Glauber, Les Houches lectures 1 $\overline{964}$, in: "Quantum Optics and Electronics," C. DeWitt et al., eds., Gordon and Breach, New York (1965);

J. R. Klauder and E. C. G. Sudarshan, "Fundamental of Quantum Optics", Benjamin, New York (1968).

4. J. M. Radcliffe, J. Phys. A 4:313 (1971).

5. A. 0. Barut and L. Girarde110, Commun. Math. Phys. 21:41 (1971).

6. A. M. Perelomov, Commun. Math. Phys. 26:222 (1972); 44:197 (1975); Usp. Fiz. Nauk 123:23 (1977) [translated into English in: Sov. Phys. Usp. 20:703 (1977)].

7. E. Onofri, J. Math. Phys. 16:1087 (1975).

8. M. M. Nieto and L. M. Simmons, Jr., Phys. Rev. Lett. 41:207 (1978);

V. P. Gutschick, M. M. Nieto and L. M. Simmons, Jr., Phys. Lett. 76A: 15 (1980); M. M. Nieto and L. M. Simmons, Jr., Phys. Rev. A 19:438 (1979) and Phys. Rev. D 20:1321,1332,1342 (1979); M. M. Nieto, Phys. Rev. D 22:391 (1980); V. P. Gutschick and M. M. Nieto, Phys. Rev. D 22:403 (1980); M. M. Nieto, I. M. Simmons, Jr. and V. P. Gutschick, Phys. Rev. D 23:927 (1981).

9. H. Feshbach and Y. Tikochinsky, Trans. N.Y. Acad. Sci. 38:44 (1977).

10. H. P. Yuen, Phys. Rev. A 13:2226 (1976).

11. D. F. Walls, Nature 306:141 (1983).

12. C. S. Hsue, H. Küme $\overline{1}$ and P. Ueberholz, Phys. Rev. D (to be published).

13. A. M. Perelomov and V. S. Popov, Zh. Eksp. Teor. Fiz. 56:1375 (1969) and 57:1684 (1969) [translated into English in: Sov. Phys. JETP 29: $7 \overline{38}$ (1969) and 30:910 (1970)].

14. S. W. Hawking, Commun. Math. Phys. 43:199 (1975).

15. P. C. W. Davies, J. Phys. A 8:609 (1975); Rep. Prog. Phys. 41:1313 (1978).

16. P. Candelas and D. J. Raine, J. Math. Phys. 17:2101 (1976).

17. W. G. Unruh, Phys. Rev. D 14:870 (1976).

18. D. W. Sciama, P. Candelas and D. Deutsch, Adv. Phys. 30:327 (1981). 